\title{
Cross-Functional Approach to Tackling Covid19 \& The Way Forward for Healthcare
}

\author{
Nadeem Ahmed N* \\ Healthcare Business Consultant and Emergency Physician, Jamshedpur, India \\ *Corresponding author: Nadeem Ahmed, Healthcare Business Consultant and Emergency Physician, Jamshedpur, India
}

\section{ARTICLE INFO}

Received: 蔧 January 21, 2021

Published: 幽 January 29, 2021

Citation: Nadeem Ahmed N. The CrossFunctional Approach to Tackling Covid19 \& The Way Forward for Healthcare. Biomed J Sci \& Tech Res 33(3)-2021. BJSTR. MS.ID.005415.

\author{
ABSTRACT
}

COVID-19 has exposed blatant gaps in health ecosystem with common issues being an excessive burden on the healthcare workers, increased morbidity and mortality among the vulnerable low-income groups and the rise of info-demic. With the wake of the second wave of the pandemic, our objective was to design a data-driven remote care model that would cater to the unaddressed mild to moderate cases with the aim to reduce the burden on healthcare workers and increase their efficiency of care delivery without compromising on patient safety. We further aimed to bolster treatment approach with an AI-based patient prioritization and an X-ray based COVID-19 screening tool powered by machine learning (ML). We implemented the model for treating patients at our private hospital and our results indicated an increase in treatment adoption by $40 \%$, patient cost-saving by $97 \%$, reduced patient acquisition cost by $59 \%$, and increase in physician satisfaction by $34.1 \%$. The X-ray based ML screening tool reduced diagnosis cost by $90 \%$ with an accuracy of $88.6 \%$ and a reduction in false-negative to $0.6 \%$. The recovered patients raised the hospital's Net promoter score to $94 \%$ from $77 \%$ baseline. With the tremendous success seen with this model, we strongly believe that the same can very well be scaled to a national level to combat the second wave of COVID-19 and that the value-added consultation approach is the way forward not only for COVID-19 but for healthcare.

\section{Introduction}

The pandemic is thus far the biggest challenge of the $21^{\text {st }}$ century, not only from a physical, mental [1] and community [2] health perspective but also from a socio-economic angle. From a purely optimistic perspective, it has helped us identify the blatant gaps in the healthcare ecosystem [3] that we would otherwise have taken years to acknowledge and maybe even a decade more to address. Although the gap analysis has provided different results for developing countries like India [4] versus a developed country like the USA [5], there is a lot of overlap.

The most significant gaps identified globally are the disproportionately low care-provider to patient ratios, the lack of essential equipment like ventilators, inefficient and sluggish allocation of essential resources and supplies to centres of care, and an overall lack of preparedness for adverse health events of such large scale. Talking from an Indian perspective, the country has just 0.8 doctors per 1000 population as against Italy's 4.1, China's 1.8, Spain's 4.1, Iran's 1.1 and the USA's 2.6. India has just 0.7 hospital beds per 1000 population as against Italy's 3.4, Spain's 3, Iran's 1.5 and the USA's 2.9 [6]. In the face of the next wave of cases, unless capacity is built quickly, more challenging times may lie ahead. Regardless of the disparity seen in the healthcare systems of different countries, certain challenges are unanimous. To name a few, the excessive burden on the healthcare workers $[7,8]$ due to inefficient resource allocation, the increased morbidity and mortality among the vulnerable low-income groups due to high out-of-pocket expense [9], and the rise of the info-demic [10,11].

\section{Objectives}

The objective was to design a data-driven remote care model (online consultation + offline support) for a private hospital that would cater to the unaddressed mild to moderate COVID19 cases in Chennai, India. The objective is further subdivided into the following three sub-objectives. The first, reduce the burden on the healthcare workers and increase their efficiency of care delivery without compromising on patient safety. The second, develop a Machine learning model for COVID19 screening, which, in association with other forms of diagnosis, would reduce the time and capital needed for diagnosis and mitigate the number of false-negative cases. And the third, provide a value-added care model that would increase 
treatment adoption, curb the spread of misinformation, and retard patients' progression to advanced disease stages.

\section{Methodology}

The study conducted over a period of 6 months started off with primary research wherein we reached out to patients $[n=25,156]$ whose contacts were available in the hospital database. The sample was further narrowed down to a target group that belonged to the low-income bracket who lived in high density or resourceconstrained settings. The final sample $[n=5,210]$ cohort consisted of patients responding to our specific queries via a survey or a telephonic interview. We were able to generate insights regarding willingness to pay for 1st online consultation and subsequent follow-ups using Van Wester drop and Garbor Granger method. The price range of 350-450 INR was found to be most suitable for the first consultation, and that of 150-250 INR was found to be apt for follow up consultations. In addition to this, our interactions helped us understand that the primary concern for our subjects with regards to the online model of care was the lack of offline support and hospitalization should such a need arise. To address this, we extended the online model to accommodate an extensive offline paramedical support team. The team took care of end-to-end needs of the patient at the comfort of their home. Their services included testing for RT-PCR, providing necessary equipment (such as pulseoximeter, $\mathrm{CBG}$ ) and medicine as per expert advice, to making routine assessments to aid physician follow-up.

To optimize the workflow, we brought in experts from the operations domain and worked alongside them to identify the supply-side limitations in handling the patient flow. We found that the most significant bottleneck was in repeat consultation, where experts were found to be overburdened with cases that did not need their level of expertise. The analysis of consultation time showed that nearly $68 \%$ of a physician's 1 st interaction with a patient was spent addressing queries and apprehensions. This insight made us on board a battery of experts ranging from a nutritionist to a counselor who could dilute the load for the physicians and help them concentrate on the patients that needed them the most.

During the study, the team developed a method of prioritization that was done both manually and with the help of an AI model that graded patient criticality, for both 1st and follow up consultation, based on the type of query, the preliminary laboratory reports, the level of anxiety, the level of comorbidity and the severity of presenting complaints. The model was further refined by taking inspiration from an artificial intelligence screening tool for COVID19 developed by the team led by Dr Andrew Soltan, an NIHR Academic Clinical Fellow at the John Radcliffe Hospital, joined with the 'AI for Healthcare' lab of Professor David Clifton within Oxford's Institute of Biomedical Engineering, and Professor David Eyre of the Oxford Big Data Institute. The tool functioned on a data set like the one employed by the in-house team for prioritization [12].
To mitigate the cost and time for diagnosis, we brought in a Machine Learning developer and provided design inputs to create an X-ray based COVID19 screening tool. To train the data set, we obtained consent for using nearly 2,500 early-stage pneumonia chest PA x-rays, from respective patients, for use in the diagnostic analysis. The deep learning architecture was built using VGG16 [13], a pre-trained deep learning model that has 16 layers of convolutional neural networks [14]. Also, we applied the transfer learning technique that was realized by using ImageNet [15] data to overcome the insufficient data and training time. To curb misinformation [16], we brought in a designer to daily infographics and short videos, ranging from 2-5 per day, in both English and vernacular language. These infographics were primarily targeted toward tackling myths and the misinformation that was taking the world by storm. Such snippets of easily digestible yet valuable lifesaving information were disseminated through YouTube, social media, and patients' contacts in our database.

\section{Results}

The dynamic consultation model increased treatment adoption by $40 \%$ in comparison with other regional hospital chains (in a radius of $7 \mathrm{kms}$ ) which catered to COVID19 patients. The valueadded consultation approach, which included a nutritionist, psychologist, and general physician, improved overall help-seeking behavior by curbing the spread of misinformation and allaying apprehensions. The online care + offline support model achieved a patient cost-saving of $97 \%$ (compared to institutional treatment of the same cadre for similar patient grades) and reduced patient acquisition cost by $60 \%$.

The X-ray based ML screening tool achieved an accuracy of $88.6 \%$ with the test data. When clubbed with the other forms of diagnosis (clinical + laboratory tests), we could mitigate falsenegative cases to near $0.6 \%$. The maximum impact was seen in patients wherein early diagnosis and treatment prevented progression of disease and thereby preventing the need for hospitalization. There was a reduction in diagnosis cost by $90 \%$, considering In-house Chest X-rays cost INR 500 versus outsourced CT-Scan, which would cost a minimum of INR 5000. Concerning time taken for diagnosis, there was a reduction in diagnosis time by over $60 \%$. This was achieved by taking a Chest X-ray on Day 3 of infection (same-day diagnosis) versus taking CT-Chest on day seven, which led to the diagnosis made on day 8 as $24 \mathrm{hrs}$ taken for report generation. As a result of the optimal mapping of patients to the right care provider, the latter could spend $\sim 59 \%$ more time in treating patients and reported an improvement in satisfaction by $34.1 \%$ over baseline levels. From a patient perspective, there was an overall improvement in health-seeking behavior as indicated by survey floated post knowledge transfer. After experiencing such a holistic care model, the recovered patients raised the hospital's Net promoter score to $94 \%$ from $77 \%$ before the implementation of the model. 


\section{Conclusion}

COVID19 has shown us that the most complex challenges, whether in healthcare or elsewhere, can be solved/handled more efficiently either through a cross-functional approach to problemsolving or implementing a sustainable business model. The Valueadded consultation approach is the way forward not only for COVID19 but for healthcare as well. The AI tool developed for patient prioritization and the Deep learning tool developed for screening COVID19 patients based on Chest PA X-Rays can very well be scaled to the national level. When extrapolated to the resource-deprived, high-volume care center, they have the power to shift provider focus from quantity to quality of care provided especially at the face of a second wave of pandemic. However, these models also have their limitations in the initial phase of development such as extension of biases of individuals who design it and the limitations of the data used to train them. Therefore, setting up a robust infrastructure for health data, which could be leveraged by people from different functions, would be vital to solving tomorrow's challenges.

\section{Financial Support and Sponsorship}

Nil.

\section{Conflicts of Interest}

There are no conflicts of interest.

\section{Author Declarations}

I confirm all relevant ethical guidelines have been followed.

\section{References}

1. Plomecka MB, Gobbi S, Neckels R, Radziński P, Skórko B, et al. (2020) Mental Health Impact of COVID-19: A global study of risk and resilience factors. medRxiv.

2. Sathian B, Asim M, Mekkodathil A, Van Teijlingen E, Subramanya SH, et al. (2020) Impact of COVID-19 on community health: A systematic review of a population of 82 million. Journal of Advances in Internal Medicine 9(1): 4-11.

\section{ISSN: 2574-1241}

DOI: 10.26717/BJSTR.2021.33.005415

Nadeem Ahmed N. Biomed J Sci \& Tech Res

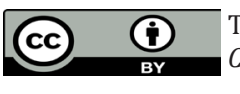

This work is licensed under Creative Commons Attribution 4.0 License

Submission Link: https://biomedres.us/submit-manuscript.php
3. Zhang H, Shaw R (2020) Identifying research trends and gaps in the context of covid-19. International journal of environmental research and public health 17(10): 3370 .

4. Chetterje P (2020) Gaps in India's preparedness for COVID-19 control. The Lancet Infectious Diseases 20(5): 544.

5. Baron A (2020) Beyond COVID19-can we heal the gaps in healthcare? International Paramedic Practice 10(2): 22-24.

6. Kamath S, Kamath R, Salins P (2020) COVID-19 pandemic in India: challenges and silver linings. Postgraduate medical journal 96(1137): 422-423.

7. Schneider M, Altersberger M, Binder C, Hengstenberg C, Binder T (2020) The COVID-19 burden for health care professionals: Results of a global survey. European Journal of Internal Medicine.

8. Erdem H, Lucey DR (2020) Health Care Worker Infections and Deaths due to COVID-9: A Survey from 37 Nations and a Call for WHO to Post National Data on their Website. International Journal of Infectious Diseases 102: 239-241.

9. El-Khatib Z, Otu A, Neogi U, Yaya S (2020) The Association between Out-of-Pocket Expenditure and COVID-19 Mortality Globally. Journal of Epidemiology and Global Health 10(3):192.

10. Kim HK, Ahn J, Atkinson L, Kahlor LA (2020) Effects of COVID-19 Misinformation on Information Seeking, Avoidance, and Processing: A Multicountry Comparative Study. Science Communication 42(5): 586615.

11. Orso D, Federici N, Copetti R, Vetrugno L, Bove T (2020) Infodemic and the spread of fake news in the COVID-19-era. European Journal of Emergency Medicine (5): 1.

12. Soltan AA, Kouchaki S, Zhu T, Kiyasseh D, Taylor T, et al. (2020) Artificial intelligence driven assessment of routinely collected healthcare data is an effective screening test for COVID-19 in patients presenting to hospital. MedRxiv.

13. Yadav S, Sandhu JK, Pathak Y, Jadhav S (2020) Chest X-ray scanning based detection of COVID-19 using deep convolutional neural network. Research square.

14. Sitaula C, Hossain MB (2020) Attention-based VGG-16 model for COVID-19 chest X-ray image classification. Applied Intelligence 17: 1-4.

15. Majeed T, Rashid R, Ali D, Asaad A (2020) Covid-19 detection using CNN transfer learning from X-ray Images. medRxiv.

16. Al-Zaman MS, Sife SA, Sultana M, Akbar M, Ahona KT, et al. (2020) Social Media Rumors in Bangladesh. Journal of Information Science Theory and Practices 8(3): 77-90.

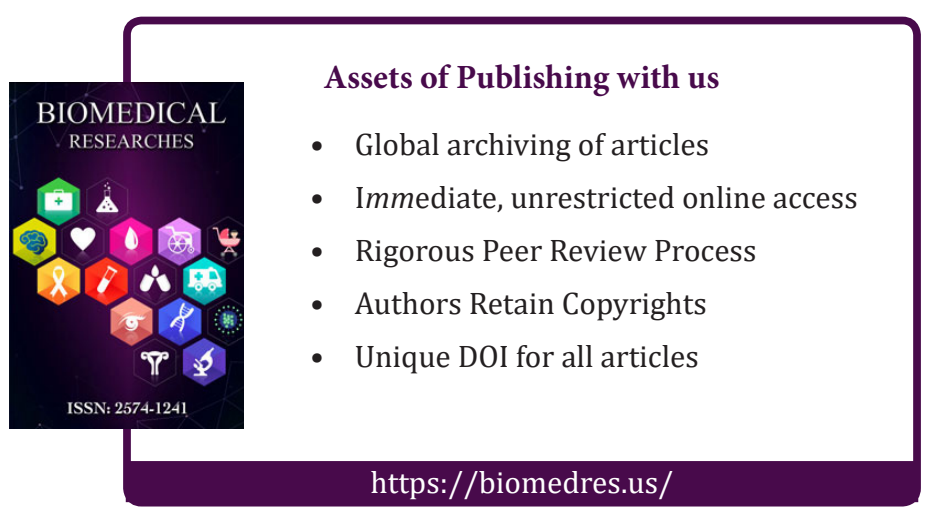

